\title{
Valores individuais e organizacionais: estudo com dirigentes de organizações pública, privada e cooperativa em Goiás
}

\author{
Kátia Barbosa Macêdo ${ }^{\mathrm{I}, 1}$, Cícero Pereira ${ }^{\mathrm{I}, 2}$, \\ Elizabeth Zulmira Rossi ${ }^{\mathrm{II}, 3}$ e Maria Aparecida Vieira ${ }^{\mathrm{III}, 4}$ \\ I'Universidade Católica de Goiás, I'Universidade Estadual de Goiás, \\ IIIUniversidade Paulista (Unip)
}

\begin{abstract}
Este artigo analisa os valores individuais dos dirigentes de três organizações (sendo uma pública, uma privada e uma cooperativa) e sua percepção sobre os valores organizacionais praticados em suas organizações. Para tanto, realizou-se um estudo numa amostra composta por 137 dirigentes de três organizações, utilizando-se como instrumentos de coleta de dados um Inventário de Valores Individuais e um Inventário de Valores Organizacionais. Após tratamento estatístico, os dados obtidos revelaram que, no que se refere aos valores individuais, para os dirigentes da organização pública, os valores da autotranscendência são os menos importantes, enquanto que, para os dirigentes das cooperativas, os valores da conservação são mais importantes como princípios que guiam suas vidas. No que se refere aos valores organizacionais, os dirigentes da organização pública percebem que os valores de conservadorismo são menos importantes para sua organização, enquanto que os dirigentes das cooperativas percebem que os valores do domínio, da autonomia, da harmonia e do igualitarismo são muito praticados nas suas organizações, sendo os valores da hierarquia os percebidos como menos praticados. As conclusões apresentam a idéia de que os valores individuais dos dirigentes, assim como os valores organizacionais, podem ser influenciados tanto pelo setor da atividade econômica ao qual a organização pertence, quanto pela cultura organizacional praticada dentro das organizações.
\end{abstract}

Palavras-chave: Valores organizacionais, Valores individuais, Organizações, Cultura organizacional.

Individual and Organizational Values: director's of Public, Private and Cooperative Organizations in Goiás

This article analyses the director's individual values of three organizations and their perception concerning the organizational values. The research had been developed with 137 directors, which work in a private, a public organization and in a cooperative, and had utilized two inventories to collect data. Data had been analysed by SPSS-Statistical Package for Social Sciences and revealed that, concerning the individual values, for the public organization director's, the autotranscendental values are the less important, and the conservational values are the most important for the directors of the cooperative. Concerning the organizational values, public organization' directors had perceived that de conservational values are less important for their organization, and the dominate, authonomy and igualitarian values are perceived for coooperative' directors as most important. The conclusion presents a discussion concerning that the individual values of the directors, even though the organizational values of the organization where they work can be influenced by the economic sector where the organization is, and by the organizational culture.

Keywords: Organizational values, Individual values, Organizations, Organizational culture.

$\mathrm{O}$ valores organizacionais têm origem na interação social entre os membros das organizações. Eles são construídos em decorrência das necessidades do trabalho a ser realizado para orientar suas relações internas e externas. Esses valores expressam a singularidade e a especificidade de cada organização, de acordo com a preferência dada a cada valor na composição de sua estrutura axiológica. Para avaliar se esses postulados apresentam algum fundamento empírico, este artigo apresenta um estudo sobre os valores individuais e organizacionais em três organizações pertencentes a setores de atividade

1 Docente dos departamentos de Administração e de Psicologia da Universidade Católica de Goiás. Doutora em Psicologia Social pela PUC-SP.

2 Docente de Psicologia Social da Universidade Católica de Goiás. Doutorando em Psicologia Social no Instituto Superior de Ciências do Trabalho (Lisboa, Portugal).

3 Docente da Universidade Estadual de Goiás. Mestre em Psicologia pela Universidade Católica de Goiás. Doutoranda em Psicologia Social e do Trabalho na Universidade de Brasília.

4 Docente da UNIP. Mestre em Psicologia pela Universidade Católica de Goiás. 
econômica diferentes. Especificamente, o estudo analisa o impacto de três organizações pertencentes a setores de atividade econômica diferentes sobre os valores individuais de seus dirigentes e na percepção que eles têm dos valores organizacionais praticados nas organizações onde atuam. Além disso, avaliam-se as possíveis relações de compatibilidade e conflito entre os valores dos dirigentes e os praticados nas organizações.

\section{Os valores individuais}

Os valores são crenças duradouras sobre formas específicas de comportamentos sociais ou sobre estados abstratos de existência (Rokeach, 1973). Recentemente, Schwartz e Bilsky (1987) identificaram princípios motivacionais básicos subjacentes aos valores individuais. Para compreender esses princípios, chamados de tipos motivacionais dos valores humanos, Schwartz e Bilsky (1990) elaboraram uma teoria que incorpora as principais características dos valores destacadas nas obras de Rokeach (1973) e de Kluckhohn (1968), definindo-os como metas individuais transituacionais que expressam interesses concernentes a um tipo motivacional. Essa definição baseou-se em três componentes considerados básicos e universais na natureza humana (Schwartz, 1994): necessidades biológicas, necessidades de interação social estável e necessidades de sobrevivência dos grupos. A partir dessas necessidades, Schwartz (1992) identificou dez tipos de valores e estabeleceu as metas motivacionais representadas por cada um deles. Para testar a universalidade das hipóteses formuladas, Schwartz (1992) elaborou um instrumento contendo 56 valores, dos quais 36 foram retirados da escala de Rokeach (1973). Schwartz (1992) verificou que na maioria das amostras foi possível identificar os dez tipos de valores hipotetizados. Essas relações são apresentadas de forma resumida na Figura 1.

Figura 1: Estrutura motivacional dos valores humanos (adaptado de Schwartz, 1992, p. 45)

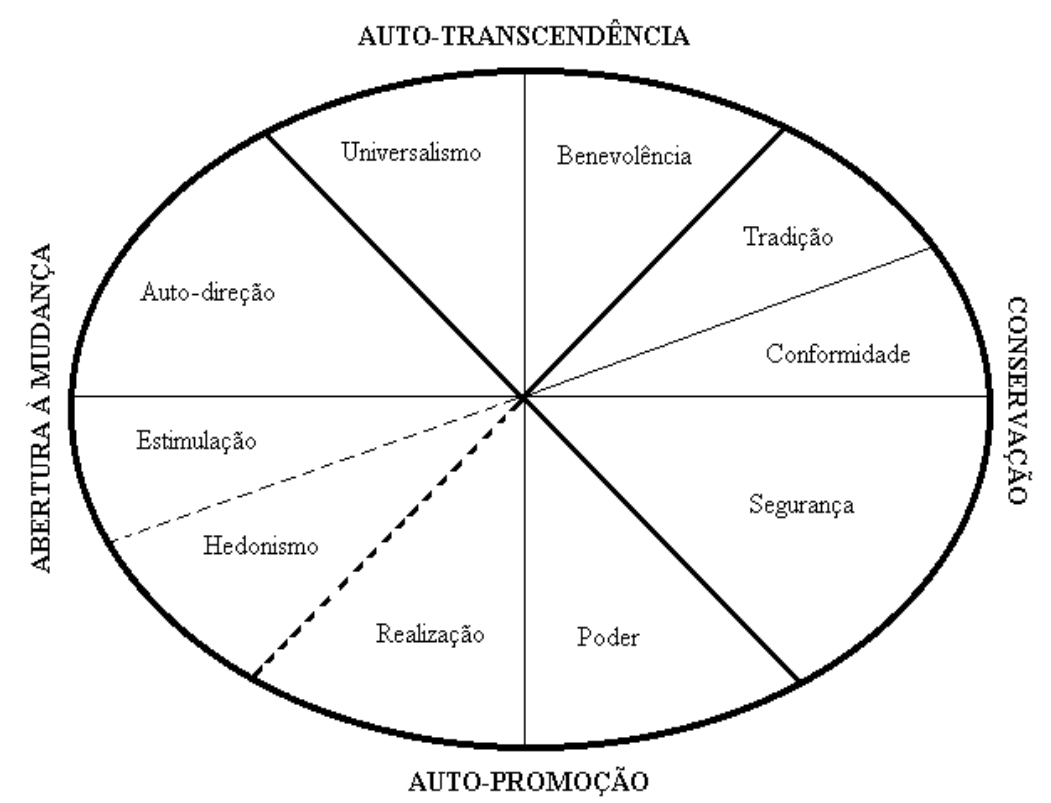

Segundo Schwartz (1992), os dez tipos de valores agrupam-se em quatro tipos de valores de ordem superior. Esses pólos são os extremos de duas dimensões valorativas em 
torno das quais situam-se os valores individuais. Uma dessas dimensões opõe os pólos da abertura à mudança aos valores que formam o pólo da conservação. O princípio que organiza essa dimensão é o conflito entre a ênfase na independência de pensamento e de ação e o favorecimento da mudança versus submissão, auto-restrição e proteção das práticas tradicionais e estabilidade pessoal. A outra dimensão opõe o pólo da autotranscendência ao pólo da autopromoção. O princípio que organiza essa dimensão é o conflito entre aceitar os outros como iguais e transcender os próprios interesses em função do bem-estar coletivo versus a busca do sucesso e o domínio sobre os outros. O hedonismo compartilha elementos tanto de abertura à mudança, quanto de autopromoção. A existência dessas dimensões foi constatada em várias culturas (Schwartz, 1994), embora algumas variações tenham sido verificadas em função do desenvolvimento cognitivo dos indivíduos (Menezes \& Campos, 1997).

Atualmente, a teoria dos tipos motivacionais é amplamente utilizada nas ciências sociais (Sagiv \& Schwartz, 2000; Schwartz \& Bardi, 2001; Tamayo \& Schwartz, 1993; Tamayo, 1994, 1996). De fato, trata-se de um modelo bem estruturado e com evidências empíricas que dão suporte a vários de seus postulados, de modo a permitir elaborar, a priori, relações hipotéticas entre os tipos motivacionais, bem como estabelecer elos entre os valores, as atitudes, as opiniões e o comportamento. Recentemente, Tamayo (1996) utilizou esse modelo para compor uma perspectiva motivacional sobre a estrutura dos valores organizacionais. Mas o que são valores organizacionais?

\section{Valores organizacionais}

Segundo Hofstede (1980), os valores constituem o núcleo da cultura organizacional. Para ele, o sistema de valores é responsável pela "programação mental", pois constituem a centralidade da cultura. Nesse sentido, valores organizacionais poderiam ser definidos como uma grande tendência nas organizações para preferir alcançar certos estados e objetivos em detrimento a outros.

Da mesma forma que Schwartz e Bilsky (1987, 1990), Hofstede (1994) concebe a estrutura de valores dentro de uma relação bipolar com características de compatibilidade e conflito. O relacionamento dos valores nas organizações não ocorre harmoniosamente. Muitas metas organizacionais mantêm vários conflitos axiológicos, tais como "liberdade" e "igualdade".

Para Tamayo (1996), a compreensão dos valores organizacionais é antecedida pelo entendimento da estrutura motivacional das pessoas. Essa estrutura, tal como a estudada por Schwartz (1992), permite identificar as dimensões organizacionais responsáveis pelos conflitos e convergências nas metas axiológicas da organização que seus trabalhadores devem incorporar. Nesse sentido, os valores organizacionais são os representantes discursivos dessas metas enfatizadas pela organização. Elas orientam as ações dos trabalhadores no ambiente organizacional. Os valores orientam as ações organizacionais porque eles são "crenças organizadas hierarquicamente, relativas a metas organizacionais desejáveis que orientassem a vida da empresa e estão ao serviço de interesses individuais, coletivos ou mistos" (Tamayo, 1996, P. 181). Neste sentido, Tamayo (1996) propõe uma estrutura dos valores organizacionais baseada na teoria dos conteúdos motivacionais dos valores humanos apresentada por Schwartz (1992). Especificamente, ele identificou três eixos axiológicos básicos que organizam os valores organizacionais. Esses eixos estão ancorados em seis pólos que reúnem os valores praticados nas organizações, os quais são organizados em função da meta motivacional que representam. A oposição entre pólos forma os seguintes eixos 
axiológicos bipolares (Tamayo, Mendes \& $\mathrm{Da} \mathrm{Paz}, 2000$ ): autonomia versus conservadorismo; hierarquia versus estrutura igualitária; e harmonia versus domínio.

De acordo com a teoria, primeiro eixo bipolar (autonomia versus conservadorismo) organiza as "relações entre o indivíduo e grupo normativo" presente no ambiente organizacional. Ela opõe os interesses próprios dos indivíduos às necessidades que as organizações têm em manter o status quo organizacional. As organizações que priorizam valores de conservadorismo enfatizam a interdição de comportamentos que possam ameaçar as normas e as tradições. As organizações que se orientam mais pelos valores de autonomia percebem o trabalhador como entidade autônoma, habilitada para perseguir os seus próprios interesses. Essas organizações valorizam tanto a autonomia intelectual quanto afetiva em seus trabalhadores. A autonomia intelectual é valorizada através da ênfase na autodeterminação. A autonomia afetiva é valorizada na prioridade dada à expressão da estimulação e do hedonismo. O segundo eixo bipolar (hierarquia versus igualitarismo) organiza as metas organizacionais em torno do conflito na "distribuição de poder" na organização. As organizações situadas no pólo de hierarquia priorizam os valores de autoridade, poder social, influência, fiscalização e supervisão. As organizações igualitárias priorizam os valores da justiça social, da igualdade, da responsabilidade e da eqüidade. $O$ terceiro eixo bipolar (harmonia versus domínio) organiza as metas motivacionais concernentes ao "relacionamento da organização com o meio físico e social". Naturalmente, os aspectos mais salientes do ambiente são os relativos às relações que as organizações mantém entre elas. Os valores pertinentes ao pólo domínio referem-se ao controle e exploração do ambiente físico e social para auto-afirmação da organização. No pólo da harmonia são encontrados os valores de proteção da natureza, de cooperação e de integração interorganizacional.

Figura 2: Estrutura motivacional dos valores organizacionais (adaptado de Tamayo, Mendes e Da Paz, 2000, p. 302)

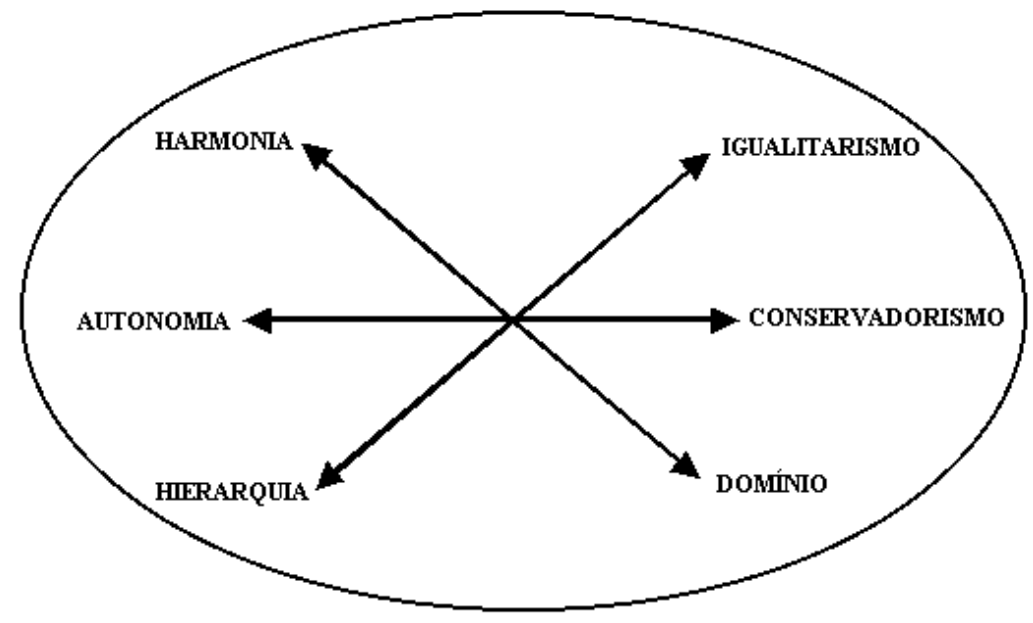

Para avaliar os três eixos axiológicos, Tamayo (1996) apresenta os fundamentos para o desenvolvimento de uma escala de medida da percepção dos valores organizacionais: Inventário de Valores Organizacionais. Esse inventário foi utilizado por Mendes (1999) em uma investigação em que os participantes da pesquisa indicaram, numa escala variando de zero a seis, o quanto eles percebem que a organização onde trabalham prioriza cada um dos 66 valores da escala. Posteriormente, Tamayo, Mendes e Da Paz (2000) identificaram os três pólos axiológicos subjacentes aos valores organizacionais ao aplicar uma Multimensional Scaling aos dados coletados em uma amostra de trabalhadores de uma empresa do Distrito Federal. Com base nesses resultados, Mendes (1999) concluiu que a compreensão do 
posicionamento das organizações nesses pólos é central ao entendimento da cultura organizacional onde as organizações estão inseridas. Nesse sentido, cabe questionar como os valores individuais e organizacionais variam conforme o posicionamento das organizações nos setores da atividade econômica. Além disso, será que, em função da organização se situar em um tipo de setor específico, os valores individuais de seus dirigentes apresentam relações de compatibilidade ou de conflito com os valores organizacionais?

\section{Os três setores da economia como contexto para se estudar os valores}

Para Borger (2001), o liberalismo, que surgiu no final do período feudal na Europa, é a doutrina econômica baseada no princípio da propriedade e da iniciativa privada que deu origem ao regime da organização atuando em um mercado livre, regido pela lei da oferta e da demanda. Na filosofia liberal, o indivíduo é considerado livre para exercer qualquer atividade econômica e dispor dos meios de produção da forma que lhe for mais eficiente para atingir o lucro. O controle é exercido pelas leis de mercado de livre concorrência. O mercado deve ser regido por suas próprias leis sem a intervenção do Estado. Nos séculos subseqüentes, o Estado, segundo esse modelo, foi considerado ineficiente e perdulário. Por essa razão, ele deveria deixar ao mercado e à sociedade civil a responsabilidade pelo desenvolvimento da nação. Neste contexto surgiram as ideologias que iriam reger as organizações livres no comércio. Essas organizações, chamadas de empresas, tinham como objetivo obter o máximo de lucro em suas atividades segundo as leis de mercado.

No século XX, o modelo econômico de cunho neoliberal foi reeditado. Para Schommer, Rocha e Fischer (1999), no contexto mundial do final do século XX, três movimentos paralelos merecem destaque. Em primeiro lugar, a crise do Estado como promotor do bem estar social abriu lacunas aos setores públicos sociais, como saúde e educação, que passaram a depender da iniciativa privada para o seu desenvolvimento e a conseqüente redução das funções estatais, a diminuição do tamanho do Estado, o equilíbrio das contas públicas e a redução dos gastos sociais (Serva,1997). Em segundo lugar, ocorreu o aumento no processo de concentração de capitais, em um cenário de economia globalizada e intensa mobilização social em nível mundial, numa onda recente de fusões e aquisições, sob a hegemonia mercado e regras de conhecimento e informação. Em terceiro lugar, as mudanças emergentes forçaram o surgimento do terceiro setor, cuja lógica é diferente da lógica do primeiro setor, o Estado, com gestão pública e fins públicos e do segundo setor, o Mercado, com gestão privada, mas fins públicos. Esse terceiro setor congrega organizações que não integram o aparelho governamental, não distribuem lucros a acionistas ou investidores e não têm tal finalidade, autogerenciam-se e possuem alto grau de autonomia interna e envolvem nível significativo de participação voluntária. Posteriormente apresentar-se-á uma melhor discussão acerca de organizações do terceiro setor, até porque não parece haver um consenso entre pesquisadores deste tema.

Nesse contexto, a sociedade passou a demonstrar seu desconforto e inquietação e promoveu o desenvolvimento de ações coletivas. Os Estados, em vários países do mundo, detectaram esta realidade emergente e perceberam nela a chave para conseguir a cooperação de que necessitavam para, sem abandonar as molduras do modelo capitalista neoliberal, ver uma sociedade construída com a participação de toda a sociedade. Para alguns autores, tratase de uma espécie de transferência de responsabilidades que antes eram prerrogativas do Estado e que, a sociedade passou a assumir pelo fato de o Estado não poder mais prover os serviços básicos à população. 
O debate sobre o conteúdo e extensão da Responsabilidade Social das Organizações nos negócios foi intenso no sentido de ir contra os princípios de lucro. Levantaram-se questões sobre a lucratividade das organizações socialmente responsáveis. Para Wood (1999), a relação entre desempenho social e financeiro é ambígua porque não há nem teoria consolidada que esclareça como devem estar relacionados, nem indicadores precisos. Segundo esse autor, a responsabilidade social organizacional não é uma restrição à maximização de lucros, mas uma variável de valor estratégico das organizações. Nesse sentido, Borger (2001) destaca que as organizações de sucesso serão, cada vez mais, pressionadas a olharem intensamente o impacto das suas operações dentro e fora de suas paredes institucionais. Cuidadosamente, elas devem verificar os impactos de suas políticas e ações nos seus trabalhadores, clientes, comunidades e na sociedade como um todo.

O contexto de transformações associado à crise do Estado e sua deficiência no suprimento de demandas sociais fizeram crescer, sob formas organizacionais variáveis, iniciativas locais que buscaram gerar novos tipos de solidariedade nos meios comunitários. Este novo espaço social é chamado de terceiro setor. Essas organizações se mobilizam para missões como a preservação do meio ambiente, a proteção dos direitos humanos, a assistência social, a defesa de minorias, além de articular a sociedade na defesa de seus direitos frente ao Estado para a fiscalização das organizações públicas e privadas e para a inserção de comunidades que vivem à margem da sociedade. $\mathrm{O}$ trabalho realizado por essas organizações solicita a criatividade e a dimensão voluntária das pessoas.

Para Wood (1999), Froes (1999), Marçon (2001) e Fernandes (1994) a expressão terceiro setor nasceu da idéia de que a atividade humana é dividida em três setores: $O$ primeiro setor, representado pelo Estado, tem o papel de estabelecer políticas públicas que atendam as reais necessidades da população, em que agentes executam ações de caráter público. O segundo setor é representado pelas organizações produtivas que compõem o mecanismo de mercado. Geralmente é o maior setor em qualquer país industrializado e os interesses privados se sobressaem aos interesses públicos. O terceiro setor é o espaço institucional que abriga ações de caráter privado, associativo e voluntarista que são voltadas para a geração de bens de consumo coletivos, sem que haja qualquer tipo de apropriação particular de excedentes econômicos que sejam gerados nesse processo (Salamon, 1998). Essas organizações complexas e diversificadas são denominadas de organizações nãogovernamentais, organizações sem fins lucrativos, organizações voluntárias, etc. Essas denominações são usadas em contextos diferentes com vários significados, causando confusão sobre o tema ainda pouco conhecido.

Segundo Thompsom (1997) e Froes (1999), as organizações do terceiro setor possuem algumas características em comum, são elas: desenvolvem atividades beneficentes, religiosas, sociais, culturais, de conhecimento e filantrópicas, objetivando concretizar as demandas e realizar ações para a finalidade social para a qual foram criadas; não possuem fins lucrativos; a partir do âmbito privado, perseguem propósito de interesse público; não integram o aparelho governamental; autogerenciam-se e gozam de alto grau de autonomia interna, descentralizando ações; envolvem nível significativo de participação voluntária e comunitária; implantam programas e projetos sociais auto-sustentáveis.

Após essa breve exposição, percebe-se que os aspectos políticos, econômicos e sociais contribuíram para a construção de vários modelos de organizações. Essas organizações, criadas dentro de uma lógica específica do setor ao qual pertencem, desenvolvem estruturas, modos de funcionamento e regulamentos específicos. Elas desenvolvem culturas internas que atendem aos objetivos da organização. Cabe, então, questionar quais são os valores praticados nessas organizações, bem como as relações desses valores com os valores das pessoas que estão à frente dessas instituições. As análises desses aspectos são particularmente importantes porque os valores são centrais na cultura organizacional (Hofstede, 1994). Portanto, realizou- 
se um estudo exploratório sobre aos valores individuais e organizacionais em três organizações pertencentes a setores da atividade econômica distintos.

\section{Metodologia da pesquisa}

Com base no conjunto de postulados teóricos apresentados, realizou-se um estudo empírico com o objetivo de analisar o impacto da posição que três organizações têm nos valores individuais de seus dirigentes e na sua percepção dos valores organizacionais praticados nas organizações onde atuam. Além disso, investigou a presença de conflitos entre os valores pessoais dos dirigentes e a sua percepção dos valores priorizados nas organizações. Nesse sentido, a investigação utilizou o método correlacional (Colman, 1995) com procedimento de coleta e análise de dados quantitativos (Pestana \& Gageiro, 2000).

\section{Os participantes}

Esta pesquisa se realizou com 137 participantes, sendo que todos ocupavam cargos de direção nas organizações onde atuavam, todas localizadas no Estado de Goiás. 80 participantes trabalhavam em uma organização pública; 26 em uma organização privada e 31 eram dirigentes de cooperativas. A idade dos participantes variou de 18 a 58 anos com média igual a 31,4 anos $(D P=10,77)$. Com probabilidade de erro menor que $5 \%$ e nível de confiança maior que $95 \%$, esse grupo pode ser considerado como uma amostra representativa de uma população estimada de 100.000 dirigentes (veja Moser e Kalton, 1972, para acesso às estratégias possíveis de amostragem).

\section{Instrumentos de coleta de dados e procedimentos de análise}

Os dados foram coletados com o Inventário de Valores Individuais (IVI) validado no Brasil por Tamayo e Schwartz (1993) e com o Inventário de Valores Organizacionais (IVO), desenvolvido e validado por Tamayo (1996) e por Mendes (1999). O IVI é um instrumento de medida dos tipos motivacionais dos valores individuais (veja Schwartz, 1992, para uma descrição mais detalhada desse instrumento).

O IVO é um instrumento composto por 66 valores que são utilizados como medida dos seis pólos dos três eixos axiológicos dos valores organizacionais. Com base nesses pólos, são estimados os posicionamentos dos indivíduos e das organizações nas dimensões que organizam os valores organizacionais. Portanto, utilizou-se esse instrumento para avaliar a percepção dos dirigentes em relação aos valores que as suas organizações priorizam (veja MENDES, 1999 para uma descrição mais detalhada desse instrumento). Os dados foram analisados no Statistical Package for Social Science (SPSS). Foram utilizadas duas técnicas estatísticas na análise dos dados: Análise Multivariada de Variâncias (MANOVA) e Correlações Parciais.

A influência do setor organizacional em que as três organizações se inserem na adesão dos dirigentes aos dez tipos motivacionais dos valores individuais e na sua percepção dos seis pólos dos valores organizacionais foi verificada através de duas MANOVAS. Para os valores individuais, essa análise foi aplicada ao delineamento 3 (organização pública, privada e cooperativa) X 10 (os dez tipos motivacionais), sendo considerados com fator a ser avaliado entre os as três organizações e os 10 tipos motivacionais como variáveis dependentes. Ainda relativamente aos valores individuais, analisou-se o impacto das organizações na adesão dos 
dirigentes aos quatro tipos motivacionais de ordem superior. A obtenção desses tipos foi acessada seguindo os critérios estabelecidos por Schwartz (1992), explicitados na Figura 1. Nesse sentido, foram identificados os quatro tipos de valores de ordem superior: autotranscendência; abertura a mudanças; autopromoção; conservação. A análise da influência das organizações também foi realizada através de uma MANOVA, considerando o delineamento 3 (pública, privada e cooperativa) X 4 (os valores de ordem superior). Para os valores organizacionais, a análise considerou o delineamento 3 (pública, privada e cooperativa) X 6 (os seis pólos dos eixos axiológicos), de modo que as três organizações foram consideradas como fatores para análise dos sujeitos e os seis pólos como variáveis dependentes. Em cada MANOVA foi utilizado o método seqüencial para soma dos quadrados, o qual oferece um ajustamento estatístico para amostras de tamanhos diferentes (veja Tabachnick e Fidell, 2001, para uma introdução à aplicação e interpretação dos resultados de uma MANOVA).

As relações entre valores individuais e percepção de valores organizacionais foram analisadas através de coeficientes de correlações parcializados. As correlações foram parciais porque se controlou sua direção e magnitude pela organização pesquisada (veja Pestana e Gageiro, 2000, para uma introdução à aplicação e interpretação das técnicas de correlação parcial). Para a realização dessas análises foi necessário estimar o posicionamento dos participantes nas dimensões dos valores individuais (Schwartz, 1992), bem como nas dimensões axiológicas dos valores organizacionais (Tamayo, 1996). A primeira dimensão dos valores individuais é autotranscendência (AT) versus autopromoção (AP). O posicionamento nessa dimensão foi estimado através da substrução entre os valores da AT e os valores da AP $(\mathrm{AT}-\mathrm{AP})$. A segunda dimensão é abertura a mudanças (AM) versus conservação $(\mathrm{CO})$. $\mathrm{O}$ posicionamento nessa dimensão foi estimado através da substrução entre os valores da AM e os itens da conservação $(\mathrm{AM}-\mathrm{CO})$. Os três eixos axiológicos dos valores organizacionais são: autonomia $(\mathrm{AU})$ versus conservadorismo $(\mathrm{CO})$; igualitarismo $(\mathrm{IG})$ versus hierarquia (HI); harmonia (HA) versus domínio (DO). O posicionamento no primeiro eixo foi obtido pela subtração entre os valores do $\mathrm{AU}$ e os itens da $\mathrm{CO}(\mathrm{AU}-\mathrm{CO})$. O posicionamento no segundo eixo foi encontrado através da subtração entre AU e os valores da HI (AU - HI). Do mesmo modo, o posicionamento no terceiro eixo foi obtido através da subtração entre os itens da HA e os valores relativos ao DO (HA - DO).

\section{Resultados}

Inicialmente, foi analisado o impacto da organização na adesão dos dirigentes aos dez tipos motivacionais dos valores individuais. Os resultados da MANOVA aplicada revelam que o efeito multivariado foi significativo: $\Lambda=0,75 ; \eta^{2}=0,14 ; F(2,131)=3,048 ; p<$ 0,05 . Esse resultado significa que a adesão dos dirigentes aos dez tipos motivacionais varia significativamente conforme a organização pesquisada, o setor da atividade econômica ao qual ela pertence.

A análise das médias de adesão a esses tipos motivacionais revelou (Tabela 1) que os dirigentes da organização privada e das cooperativas aderem mais fortemente aos valores de universalismo do que os dirigentes da organização pública. Com relação aos tipos motivacionais de benevolência e de autodireção, os dirigentes das cooperativas aderem mais a esses valores do que os dirigentes da organização pública. Já no que se refere aos tipos motivacionais da conformidade e da tradição, os dirigentes das cooperativas aderem mais a esses do que os dirigentes da organização privada e os dirigentes da organização pública. 
Tabela 1: Médias de adesão aos tipos motivacionais dos valores individuais em função da organização

\begin{tabular}{llll}
\hline & \multicolumn{2}{l}{ Organização } & \\
\cline { 2 - 4 } Tipos de Valores & Pública & Privada & Cooperativa \\
\hline Universalismo & $4,4_{\mathrm{b}}$ & $4,8_{\mathrm{a}}$ & $4,9_{\mathrm{a}}$ \\
Benevolência & $4,8_{\mathrm{b}}$ & $5, \mathrm{O}_{\mathrm{ab}}$ & $5,2_{\mathrm{a}}$ \\
Autodireção & $4,6_{\mathrm{b}}$ & $4,7_{\mathrm{ab}}$ & $5,0_{\mathrm{a}}$ \\
Estimulação & 2,9 & 3,0 & 3,1 \\
Hedonismo & 4,6 & 4,3 & 4,4 \\
Poder & 3,3 & 3,1 & 3,5 \\
Auto-realização & 4,3 & 4,4 & 4,5 \\
Segurança & 4,6 & 4,4 & 4,8 \\
Conformidade & $4,5_{\mathrm{b}}$ & $4,7_{\mathrm{b}}$ & $5,1_{\mathrm{a}}$ \\
Tradição & $3,8_{\mathrm{b}}$ & $3,7_{\mathrm{b}}$ & $4,1_{\mathrm{a}}$ \\
\hline
\end{tabular}

Nota: As médias que não compartilham o mesmo subscrito representam diferenças estatisticamente significativas no teste de Duncan $\operatorname{com} p<0,05$.

Após a análise do impacto das três organizações nos tipos motivacionais de primeira ordem, investigou-se o impacto dessas organizações na adesão dos dirigentes aos quatro tipos motivacionais de ordem superior. Os resultados da MANOVA mostraram que o efeito multivariado dessa análise foi significativo, o que indica que a adesão dos dirigentes aos valores de ordem superior variou em função de trabalharem em uma organização específica: $\Lambda=0,88 ; \eta^{2}=0,06 ; F(2,131)=2,128 ; p<0,05$. Essa influência ocorreu na adesão aos tipos motivacionais da autotranscendência e da conservação. A análise das médias de adesão a esses tipos motivacionais mostrou (Tabela 2) que os dirigentes da organização pública são os que menos valorizam os valores da autotranscendência e os dirigentes da cooperativa são os que mais valorizam os componentes da conservação.

Tabela 2: Médias de adesão aos valores de ordem superior em função das organizações

\begin{tabular}{llll}
\hline & \multicolumn{2}{l}{ Organização } & \\
\cline { 2 - 4 } Tipos de Valores & Pública & Privada & Cooperativa \\
\hline Autotranscendência & $4,6_{\mathrm{b}}$ & $4,9_{\mathrm{a}}$ & $5,1_{\mathrm{a}}$ \\
Abertura à Mudança & $4,0_{\mathrm{a}}$ & $4,0_{\mathrm{a}}$ & $4,2_{\mathrm{a}}$ \\
Autopromoção & $3,8_{\mathrm{a}}$ & $3,8_{\mathrm{a}}$ & $4,0_{\mathrm{a}}$ \\
Conservação & $4,3_{\mathrm{b}}$ & $4,3_{\mathrm{b}}$ & $4,7_{\mathrm{a}}$
\end{tabular}

Nota: As médias que não compartilham o mesmo subscrito representam diferenças estatisticamente significativas segundo o teste de Duncan $\operatorname{com} p<0,05$.

No que se refere à influência das organizações na percepção que os dirigentes tinham dos valores praticados nas organizações, o efeito multivariado da MANOVA permitiu constatar que, de um modo geral, o setor organizacional a que pertence uma organização influencia a percepção dos valores organizacionais: $\Lambda=0,39 ; \eta^{2}=0,37 ; F(2,131)=23,289$; $p<0,001$. A análise das médias de adesão aos seis pólos dos valores organizacionais (Tabela 3) revelou que os dirigentes da organização privada percebiam que a organização onde atuavam valorizava menos os valores de conservação do que os dirigentes da organização 
pública e da cooperativa. Os gerentes da cooperativa percebia que a organização valorizava mais que os dirigentes da organização pública e privada os valores do domínio, da autonomia, da harmonia, e do igualitarismo, ao passo que percebiam que a organização aderia menos aos valores da hierarquia.

Tabela 3: Médias de percepção dos tipos motivacionais dos valores organizacionais em função das organizações

\begin{tabular}{|c|c|c|c|}
\hline \multirow[b]{2}{*}{ Tipos de Valores } & \multicolumn{3}{|c|}{ Organização } \\
\hline & Pública & Privada & Cooperativa \\
\hline Conservadorismo & $4,6_{a}$ & $4,1_{\mathrm{b}}$ & $4,7_{\mathrm{a}}$ \\
\hline Domínio & $3,3_{\mathrm{b}}$ & $3,5_{\mathrm{b}}$ & $4,7_{\mathrm{a}}$ \\
\hline Autonomia & $3,6_{b}$ & $3,5_{\mathrm{b}}$ & $4,4 a$ \\
\hline Harmonia & $3,2 \mathrm{~b}$ & $2,9_{\mathrm{b}}$ & $4,3_{a}$ \\
\hline Hierarquia & $4,7_{\mathrm{a}}$ & $4,3_{\mathrm{ab}}$ & $4,1_{\mathrm{b}}$ \\
\hline Igualitarismo & $3,1_{\mathrm{b}}$ & $2,7_{\mathrm{b}}$ & $4,1_{\mathrm{a}}$ \\
\hline
\end{tabular}

Nota: As médias que não compartilham o mesmo subscrito representam diferenças estatisticamente significativas no teste de Duncan $\operatorname{com} p<0,05$.

No que concerne às relações entre valores individuais e valores organizacionais, a Tabela 4 apresenta os resultados das correlações parciais calculadas entre as dimensões motivacionais dos valores individuais e as dimensões axiológicas dos valores, controladas pelas três organizações. Como pode ser constatado, os resultados mostram padrões correlacionais distintos em função das organizações. Na organização pública as correlações eram positivas, indicando ausência de conflitos axiológicos. Na organização privada, as correlações revelaram a existência de conflitos axiológicos generalizados. Como pode ser observado, todas as correlações entre os valores individuais e organizacionais eram negativas. $\mathrm{Na}$ cooperativa apenas duas correlações eram significativas.

Tabela 4: Coeficientes de correlações parciais entre valores individuais e percepção de valores organizacionais controladas pelas organizações

\begin{tabular}{|c|c|c|c|c|c|c|c|c|c|c|c|}
\hline & \multicolumn{11}{|c|}{ Organização } \\
\hline & \multicolumn{4}{|c|}{ Pública } & \multicolumn{4}{|c|}{ Privada } & \multicolumn{3}{|c|}{ Cooperativa } \\
\hline & \multicolumn{2}{|c|}{$\mathrm{AT}-\mathrm{AP}$} & \multicolumn{2}{|c|}{$\mathrm{AM}-\mathrm{CO}$} & \multicolumn{2}{|c|}{$\mathrm{AT}-\mathrm{AP}$} & \multicolumn{2}{|c|}{$\mathrm{AM}-\mathrm{CO}$} & \multicolumn{2}{|c|}{$\mathrm{AT}-\mathrm{AP}$} & \multirow{2}{*}{$\begin{array}{l}\text { AM-CO } \\
0,11\end{array}$} \\
\hline $\mathrm{AU}-\mathrm{CO}$ & 0,27 & $* *$ & 0,24 & $*$ & $-0,42$ & $* *$ & $-0,43$ & $* *$ & 0,27 & $*$ & \\
\hline IG-HI & 0,16 & & 0,16 & & $-0,48$ & $* * *$ & $-0,43$ & $* *$ & 0,19 & & $-0,12$ \\
\hline HÁ-DO & 0,20 & $*$ & 0,11 & & $-0,43$ & $* *$ & $-0,26$ & & $-0,43$ & $* * *$ & $-0,15$ \\
\hline
\end{tabular}

Nota: A dimensão AT-AP varia de 8 (maior adesão à autotranscendência) a - 8 (maior adesão à autopromoção). A dimensão AM-CO varia de 8 (maior adesão à abertura a mudanças) a - 8 (maior adesão à conservação). $\mathrm{O}$ eixo AU-CO varia de 6 (maior percepção de autonomia) a - 6 (maior percepção de conservadorismo). O eixo IG-HI varia de 6 (maior percepção dos valores do igualitarismo) a - 6 (maior percepção de hierarquia). O eixo HA-DO também varia de 6 (maior percepção de harmonia) a -6 (maior percepção de domínio). *p<0,05; ** $p<0,01 ; * * p<0,001$. 


\section{Discussão}

É importante comentar que por tratar-se de uma pesquisa de caráter exploratório, não houve desde o princípio nenhuma intenção de se generalizar os dados aqui trabalhados, considerando as limitações metodológicas do estudo apresentado. No entanto, cabe ressaltar que os resultados obtidos podem sim servir de indicadores para a construção do conhecimento que aborda a relação entre cultura, organizações e valores. Assim, pode-se afirmar que os resultados obtidos apontaram para a influência dos setores de atividade econômica a que pertencem as organizações nos valores individuais de seus dirigentes e ainda nos valores percebidos por eles como priorizados pelas organizações onde atuam. A forma como ocorre a interferência dos setores nas organizações, nos valores individuais e organizacionais difere, talvez em decorrência das especificidades da cultura de cada organização pesquisada, uma vez que a cultura organizacional é construída a partir da história, a missão, os objetivos e as metas os processos, as interações sócioprofissionais. Em decorrência destes fatores de ordem estrutural, os valores adotados como prioritários pelas organizações também se diferenciam.

A análise dos valores individuais foi realizada com base na organização dos tipos motivacionais de valores em uma estrutura bipolar (Schwartz, 1992). A análise dos valores com base nessa estrutura permitiu verificar os efeitos do processo levado a cabo pelas organizações, que consiste em promover para cargos gerenciais e diretivos, os trabalhadores que demonstram consonância com os valores de sua cultura. De fato, os resultados mostraram que há valores relacionados com a cultura de cada organização, de acordo com o setor ao qual pertence. Assim, não se pode afirmar que é o fato de a organização pertencer a um determinado setor que determina os valores individuais, porém pode-se inferir que quando ocorre consonância entre os valores individuais e os organizacionais, pode haver mais chances de a pessoa ser contratada ou mesmo promovida na organização.

Nesse sentido, os dirigentes da organização pública foram os que consideraram menos importantes os valores cujas metas motivacionais eram a compreensão, proteção da natureza e bem-estar de todos. Isto pode ser explicado pelo fato de que se trata de uma organização que enfatiza os princípios organizadores da hierarquia, do controle de normas e procedimentos, sendo que valores como compreensão e bem-estar de todos não poderiam mesmo ter prioridade. O lema da organização é que seus trabalhadores devem se colocar a serviço da sociedade, promover segurança à população, desenvolvendo políticas de recursos humanos de caráter instrumental. A ausência de conflitos axiológicos na organização pública indica que quanto mais os dirigentes valorizam a autopromoção e a conservação, mais fortalecida é a sua percepção de que a organização onde atuam prioriza o conservadorismo e o domínio. Assim, os valores cumprem sua função na percepção dos dirigentes de atender aos objetivos organizacionais e as necessidades das pessoas, tal como destacado por Mendes (1999).

$\mathrm{Na}$ organização privada, ao contrário dos dirigentes da organização pública, as correlações mostraram conflitos axiológicos generalizados. Nesse sentido, quanto mais importantes eram os valores de autotranscendência e de abertura a mudanças, menor era o grau de percepção de que a organização que eles dirigiam valorizava a autonomia, o igualitarismo e a harmonia. Essas características podem ocorrer como resposta pela exigência de que a organização voltada para o mercado deve ser flexível para se adaptar às mudanças, com foco específico no cliente externo. Assim, os valores dos trabalhadores devem ser relevados a um segundo plano, pois "o cliente tem sempre razão". A identificação desses conflitos na cultura organizacional é particularmente importante no diagnóstico de problemas de auto-estima, de insatisfação no trabalho (Meglino, Ravlin \& Adkins, 1989; Shoskley \& Morley, 1989) e, conseqüentemente, de produção dos trabalhadores. Assim, trabalhadores que percebem os valores organizacionais em dissonância com seus valores individuais tendem 
a não se automotivarem para perseguir os objetivos desta organização, o que leva a uma suposta baixa produtividade (Chiavenato, 2001; Vergara, 2000; Vroom, 1997).

Os dirigentes da cooperativa foram os que mais valorizaram a manutenção do status $q u o$, o fortalecimento dos costumes culturais e religiosos, o controle dos comportamentos dissonantes em relação às normas sociais. À primeira vista, pode parecer um contra-senso para uma cooperativa valorizar conservadorismo e domínio, pois parece ser esses valores próprios das organizações públicas e privadas, como está preconizado pela teoria (Tamayo, 1996). Porém, a adesão aos valores de autonomia, harmonia e igualitarismo significa um contraponto na organização dessa estrutura motivacional dos valores organizacionais. As características que identificam as cooperativas envolvem o trabalho voluntário (comunitário e não lucrativo), a preocupação com o meio ambiente e com os direitos humanos, um grau alto de autonomia, distribuição do poder e autogerenciamento (Borger, 2001; Thompsom, 1997).

Essas interpretações são refletidas nas correlações que indicam tanto existência quanto a ausência de conflitos axiológicos na cooperativa. De fato, quanto mais os dirigentes valorizam a autotranscendência maior é o grau de percepção que eles têm de que as suas organizações valorizam a autonomia. Contudo, também quanto maior é a importância da autotranscendência como princípio guia na vida dos dirigentes, maior é a percepção de que as organizações que eles dirigem priorizam os valores do domínio. É importante notar que a ausência de conflito entre os tipos de valores individuais e valores organizacionais refere-se à dimensão que estrutura as relações interpessoais na organização. Já os conflitos ocorrem em relação à dimensão que organiza as estratégias que as organizações utilizam para se relacionarem com as outras organizações.

No que se refere aos valores organizacionais, os dados mostraram que para os dirigentes da organização pública, a organização valorizava a manutenção do status quo organizacional, expressa no controle de comportamentos que pudessem ameaçar as normas sociais e as tradições. Os dirigentes da cooperativa perceberam mais fortemente que os dirigentes das outras organizações, que sua organização priorizava os valores da justiça social, da igualdade, da responsabilidade da eqüidade na distribuição do poder na organização. Eles também perceberam que sua organização valorizava a capacidade que o trabalhador tinha de seguir de forma autônoma seus interesses intelectuais e afetivos. Além disso, eles perceberam,mais que os outros dirigentes, a valorização da proteção da natureza, de cooperação e de integração interorganizacional. Os dirigentes da cooperativa perceberam que sua organização também priorizava o controle e exploração do ambiente físico e social para sua auto-afirmação. Já os dirigentes das organizações pública e privada perceberam que suas organizações priorizavam os valores da autoridade, do poder social, da influência, da fiscalização e da supervisão.

Após a discussão dos dados, pode-se afirmar que os dados coletados no presente estudo de caso permitem inferir que a organização de uma estrutura motivacional de valores organizacionais pode estar relacionada com o setor econômico a que as organizações se inserem. Como afirmam Tamayo (1996) e Mendes (1999), os valores organizacionais são representantes das metas enfatizadas e orientam as ações, as percepções, as visões, a construção da subjetividade no ambiente organizacional. Neste sentido, os valores individuais e organizacionais são priorizados mais ou menos conforme o setor da atividade econômica a que a organização pertence, tendo em vista os valores que estas organizações adotam para a constituição de sua cultura organizacional, que são percebidos pelos seus trabalhadores. Assim, pode-se supor que os valores adotados na constituição da cultura organizacional podem nortear a escolha de seus trabalhadores, desde a contratação até a definição de critérios de promoção para cargos gerenciais ou diretivos. Essas suposições podem ser fonte de hipóteses passiveis de teste em outros estudos. 


\section{Referências}

Borger, F. G. (2001). Responsabilidade social: efeitos da atuação social na dinâmica empresarial. Tese de Doutorado, Faculdade de Economia, Administração e Contabilidade, Universidade de São Paulo, São Paulo.

Chiavenato, I. (2001). Gestão de Pessoas. São Paulo: Atlas.

Colman, A. M. (1995). Psychological research methods and statistics. New York: Longman.

Drucker, P. (1999). Administrando em tempos de grandes mudanças. São Paulo: Pioneira.

Fernandes, C. R. (1994). Privado, porém público: o terceiro setor na América Latina. São Paulo: RelumeDumará.

Schommer, P. C., Rocha, A. G. P. \& Fischer, T. (1999). Cidadania empresarial no Brasil: três organizações baianas entre o mercado e o terceiro setor. In Anais do Encontro Nacional da Associação Nacional de Pós-Graduação em Administração. Foz do Iguaçu: Anpad.

Froes, C. (1999). Responsabilidade social e cidadania empresarial: a administração do terceiro setor. Rio de Janeiro: Qualitymark.

Hofstede, G. (1980). Culture's consequences. Beverly Hills: Sage.

Hofstede, G. (1994). Cultures and organizations: cultural cooperation and its importance for survival. London: McGraw Hill.

Kluckhohn, C. (1968). Los valores y las orientaciones de valor en la teoría de la acción. In T. Parsons \& E. A. Shils (Orgs.). Hacia una teoría general de la acción (pp. 435-485). Buenos Aires: Editorial Kapelusz.

Marçon, D. (2001). Gestão das organizações do terceiro setor: um repensar sobre as teorias organizacionais. In Anais do Encontro Nacional da Associação Nacional de Pós-Graduação e Pesquisa em Administração. Campinas: Anpad.

Meglino, M. B., Ravlin, C. \& Adkins, C. (1989). A work values approach to corporate cultures: a field test of the values congruence process and its relationship to individual outcomes. Journal of Applied Psychology, 74 (3), 424-432.

Mendes, A. M. (1999). Valores e vivências de prazer-sofrimento no contexto organizacional. Tese de Doutorado, Instituto de Psicologia, Universidade de Brasília, Brasília.

Menezes, I. \& Campos, B. (1997). The process of value-meaning construction: a cross-sectional study. European Journal of Social Psychology, 27 (1), 55-77.

Moser, C. A. \& Kalton, G. (1972). Survey methods in social investigation. New York: Basic Books.

Pestana, M. H. \& Gageiro, J. N. (2000). Análise de dados para ciências sociais: a complementaridade do SPSS. Lisboa: Edições Silabo.

Rokeach, M. (1973). The nature of human values. New York: Free Press.

Sagiv, L. \& Schwartz, S. H. (2000). Value priorities and subjective well-being: Direct relations and congruity effects. European Journal of Social Psychology, 30 (1), 177-198.

Salamon, E. (1998). A emergência do terceiro setor: uma revolução associativa global. Revista de Administração de Empresas, São Paulo, 33 (1), 5-11.

Schwartz, S. H. (1992). Universals in the content and structure of values: theoretical advanced and empirical testes in 20 countries. In M. Zanna (Org.), Advances in experimental social psychology (pp. 1-65). Orlando: Academic Press.

Schwartz, S. H. (1994). Are there universal aspects in the structure and contents of human values? Journal of Social Issues, 50 (1), 19-45.

Schwartz, S. H. \& Bardi, A. (2001). Value hierarchies across cultures: Taking a similarities perspective. Journal of Cross-Cultural Psychology, 32 (2), 268-290. 
Schwartz, S. H. \& Bilsky, W. (1987). Toward a universal psychological structure of human values. Journal of Personality and Social Psychology, 53 (3), 550-562.

Schwartz, S. H. \& Bilsky, W. (1990). Toward a theory of the universal content structure of values: Extensions and cross-cultural replications. Journal of Personality and Social Psychology, 58 (4),878891.

Serva, M. (1997). O Estado e as ONGs: uma parceria complexa. Revista de Administração Pública, 31 (6), 41-54.

Shoskley, E. \& Morley, D. (1989). Adhering to organizational culture. What is mean? Why does it matter? Group E Organizational Studies, 14 (4), 483-500.

Tabachnick, B. G. \& Fidell, L. S. (2001). Using multivariate statistics. Boston: Allyn \& Bacon.

Tamayo, A. (1994). Hierarquia dos valores transculturais e brasileiros. Psicologia: Teoria e Pesquisa, 10 (2), 269-285.

Tamayo, A. (1996). Cultura, valores organizacionais. In A. Tamayo, J. E. Borges \& W. Codo (Orgs.), Trabalho e cultura (pp. 157-172). São Paulo: Cooperativa de Editores Associados.

Tamayo, A. \& Schwartz, S. H. (1993). Estrutura motivacional dos valores humanos. Psicologia: Teoria e Pesquisa, 9 (2), 328-346.

Tamayo, A., Mendes, A. M. \& Da Paz, M. G. T. (2000). Inventário de valores organizacionais. Estudos de Psicologia, 5 (2), 289-315.

Thompsom, A. A. (1997). Do compromisso à eficiência? Os caminhos do terceiro setor na América Latina. In E. Ioschpe (Org.), Terceiro setor: desenvolvimento social sustentado (pp. 89-128). Rio de Janeiro: Paz e Terra.

Vergara, S. C. (2000). Gestão de Pessoas. São Paulo: Atlas.

Vroom, V. H. (1997). Gestão de pessoas, não de pessoal. Rio de Janeiro: Campus.

Wood, J. R. T. (1999). Abc da terceira via. Carta Capital, 6 (93), 68.

Endereço para correspondência: katia.macedo@cultura.com.br 